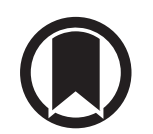

CrossMark

\section{QuantiFERON test interpretation in patients receiving immunosuppressive agents: an alert}

To the Editor:

Latent tuberculosis infection (LTBI) diagnosis in immune-mediated inflammatory diseases is complex because of the patient characteristics and the imperfection of available tests [1]. Functional T-cell assays are exposed to bias at several levels (manufacturing, pre-analytical, analytical and immunological) [2] and, unfortunately, the lack of a gold standard precludes higher diagnostic accuracy. However, the QuantiFERON GOLD In-tube test (QFT; QIAGEN, Hilden, Germany) represents a largely used and modern alternative to the tuberculin skin test. Even if performance specifications of QFT are of great interest (specificity of $99 \%$ and sensitivity of $84 \%$ ) [3], some patients remain with an "indeterminate result" because of failed positive control corresponding to the incapacity of lymphocytes to secrete interferon (IFN) $\gamma$ after $24 \mathrm{~h}$ of stimulation by phytohemagglutinin A (PHA).

The manufacturer indicates that indeterminate results occur when both nil and antigen-stimulated sample wells are negative and if the value of the positive control well (response to mitogen) is less than $0.5 \mathrm{IU} \cdot \mathrm{mL}^{-1}$ after subtraction of the value of the nil well. The clinical relevance of this $0.5 \mathrm{IU} \cdot \mathrm{mL}^{-1}$ threshold remains to be clarified. In strict respect of pre-analytical procedures, the response to PHA relies on the functional integrity of lymphocytes, which can be altered in various clinical contexts. First, the reported prevalence of indeterminate results is higher in patients (up to 29\% [4]) than in healthy people (1.5\%) [5]. Secondly, independent predictors of indeterminate QFT have been described that deal with immunological parameters. For example, patients with chronic inflammatory conditions (high neutrophil to lymphocyte ratio) have been described as more likely to have indeterminate QFT than healthy subjects [6]. Otherwise, patients receiving at least one immunosuppressive drug (especially steroids) are more likely to have an indeterminate result $[1,4,7,8]$. We made the hypothesis that the proportion of "bad" responders to mitogen among patients with negative QFT could be underestimated, especially during immunosuppressive drug intake.

The aim of our study was to determine the proportion of "bad" responders to mitogen among patients with negative QFT and to discuss the clinical relevance of the $0.5 \mathrm{IU} \cdot \mathrm{mL}^{-1}$ threshold for positive control.

Patients tested with QFT in the immunology laboratory of the University Hospital of Toulouse, France, between August 2015 and August 2016 were retrospectively enrolled. QFT-Gold tests were prescribed by hospital physicians for inpatients or outpatients of any ward. On the request form, prescribing physicians reported treatment with IS. In total, 3167 requests were sent to the laboratory. A total of 286 requests were excluded because the laboratory procedure was not valid, and 897 were excluded because the treatment field was not fulfilled by the clinicians. Finally, 1984 workable requests were kept for further analysis. Arbitrarily, responses to PHA were distinguished in four categories: no response (IFN- $\gamma<0.5 \mathrm{IU} \cdot \mathrm{mL}^{-1}$ ), bad response (IFN- $\gamma$ 0.5-5 IU.mL $\mathrm{mL}^{-1}$ ), low response (IFN- $\gamma$ 5-10 IU.mL ${ }^{-1}$ ) and normal response (IFN- $\gamma$ $>10 \mathrm{IU} \cdot \mathrm{mL}^{-1}$ ). Immunosuppressive agents encompassed a large number of drugs, mainly represented by anti-tumour necrosis factor (TNF)- $\alpha$, anti-retroviral therapy (ARV), azathioprine (AZT), methotrexate (MTX), mycophenolate mofetil (MMF), non-steroidal anti-inflammatory drugs (NSAIDs), rituximab and steroids. Patients receiving other medications, not directly interfering with the immune system, were classified in the "no immunosuppressives" group.

@ERSpublications

Caution to patients with negative QuantiFERON and low mitogen response receiving immunosuppressive agents http://ow.ly/MKsP308O3jo

Cite this article as: Belliere J, Blancher A. QuantiFERON test interpretation in patients receiving immunosuppressive agents: an alert. Eur Respir J 2017; 49: 1602102 [https://doi.org/10.1183/13993003.021022016]. 
In our cohort, results for QFT were found to be positive in $12.2 \%$ patients, negative in $82.5 \%$ patients and indeterminate in $5.3 \%$ patients. As expected, immunosuppressive intake led to more indeterminate results ( $10 \%$ versus $3 \%)$. Moreover, immunosuppressive intake was associated with a reduced proportion of positive QFT (only $8 \%$ versus $14 \%$ in the "no immunosuppressives" group). A similar result has been recently reported in immunocompromised patients [9], suggesting a possible excess of false-negative QFT by unknown altered response to tuberculosis antigens.

Hereafter we report on responses to PHA in patients with indeterminate or negative QFT.

First, we addressed the impact of immunosuppressive drug intake on response to PHA. No responders (defined with the consensual $0.5 \mathrm{IU} \cdot \mathrm{mL}^{-1}$ threshold) encompassed a majority (63\%) of patients receiving immunosuppressives, mainly steroids alone or in association (46\%). Bad responders, defined by the IFN- $\gamma$ $<5 \mathrm{IU} \cdot \mathrm{mL}^{-1}$ threshold, were much more frequent in the group of subjects receiving immunosuppressive agents than in the "no immunosuppressives" group $(22 \%$ versus $14 \%$, Fisher's exact test $\mathrm{p}<0.0001)$. Low responders, defined by the IFN- $\gamma<10 \mathrm{IU} \cdot \mathrm{mL}^{-1}$ threshold, were also much more frequent in the group of subjects receiving immunosuppressive agents group than in the "no immunosuppressives" group ( $47 \%$ versus $31 \%$, Fisher's exact test $\mathrm{p}<0.0001)$. Thus, immunosuppressive agent intake strongly affected the response to PHA.

Secondly, we wondered whether the type of immunosuppressive agent had an impact on response to PHA. As depicted in figure 1, responses to mitogen were absolutely not homogeneous above the arbitrary $0.5 \mathrm{IU} \cdot \mathrm{mL}^{-1}$ threshold: bad responders (IFN- $\gamma<5 \mathrm{IU} \cdot \mathrm{mL}^{-1}$ ) represented a variable but significant proportion of patients (14-53\%), depending upon the nature of immunosuppressive. Although some treatments did not affect response to mitogen (e.g. anti-TNF, ARV, NSAID), the proportions of "bad responders" were significantly higher in patients receiving the following treatments than in controls $(17 \%)$ : AZT (50\%, p<0.0001), MMF (53\%, p=0.0017), MTX (32\%, p=0.0003) or steroids $(43 \%, \mathrm{p}<0.0001)$, using Fisher's exact tests. Interestingly, the effects of AZT, MMF, MTX and steroids on response to mitogen were still significant even if raising the threshold from $5 \mathrm{IU} \cdot \mathrm{mL}^{-1}$ to $10 \mathrm{IU} \cdot \mathrm{mL}{ }^{-1}$ to define "low responders".

Finally, we investigated the IFN- $\gamma$ release of 254 healthy individuals (healthy blood donors, sex ratio 1; age 20-65 years; free of contact with tuberculosis patients) after $24 \mathrm{~h}$ of stimulation by PHA of whole blood samples. The proportion of low responders (IFN- $\left.\gamma<10 \mathrm{IU} \cdot \mathrm{mL}^{-1}\right)$ was very low $(\mathrm{n}=3 ; \sim 1 \%)$, similar to that previously reported in healthy individuals screened by means of QuantiFERON test. Surprisingly, the geometric mean of IFN- $\gamma$ concentration was around $120 \mathrm{IU} \cdot \mathrm{mL}^{-1}$, much higher than $10 \mathrm{IU} \cdot \mathrm{mL}^{-1}$, the maximum concentration of IFN- $\gamma$ which is measurable by the QuantiFERON test.

Indeterminate results still represent a thorny problem in QFT interpretation. Reported rates of indeterminate QFT results depend on immunosuppressive use in presented populations, and vary from $4 \%$ [10], $5 \%$ [7], $10 \%$ [11], 21\% [12] to $29 \%$ [4]. Our 5\% rate is coherent, given that our cohort was selected without disease-related inclusion criteria. We then demonstrated that responses to mitogen were highly variable and strongly affected by AZT, MMF, MTX and steroid intake. Previous studies had already reported similar results on steroids, MTX and AZT but with far smaller effectives and on selected cohorts with inflammatory bowel and rheumatologic diseases [4, 7]. To our knowledge, the role of MMF has not been reported to date. In a recent work, SESTER et al. [9] described responses to QFT in immunocompromised patients and showed that indeterminate QFT frequency was related to the extent of immunosuppression. The reported percentages of indeterminate results were $1.9 \%$ in controls (versus $1 \%$ in our cohort), and rose to $20.3 \%$ in patients with solid organ transplantation. A high drug score (obtained

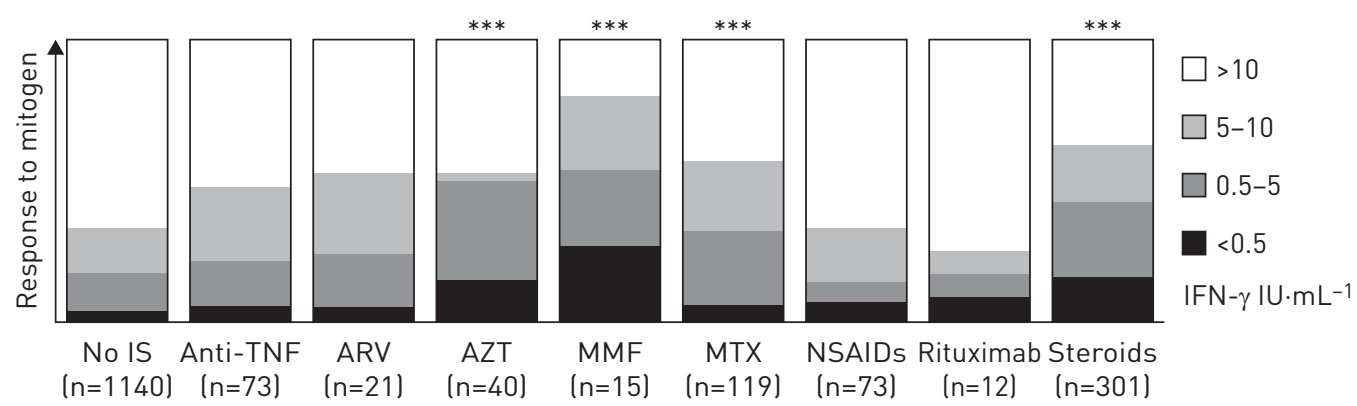

FIGURE 1 Altered responses to mitogen in patients with a negative QFT-Gold In-tube test assay receiving immunosuppressive agents. "No IS" corresponds to patients receiving medications not directly interfering with the immune system. Anti-TNF: anti-tumour necrosis factor- $\alpha$; ARV: anti-retroviral therapy; AZT: azathioprine; MMF: mycophenolate mofetil; MTX: methotrexate; NSAID: non-steroidal anti-inflammatory drug. ${ }^{* * *}: p<0.001$ with Fisher's exact test (patients with response to mitogen had a value between 0 and $5 \mathrm{IU} \cdot \mathrm{mL}^{-1}$; treated group versus "no IS" group). 
with IS combination, including for example steroids, AZT, MMF) was associated with a higher percentage of indeterminate QFT [9], consistent with our findings underlying the role of these medications.

In conclusion, we provided evidence indicating that the threshold for indeterminate results should be adapted to the studied population. According to the data obtained on healthy individuals, a response to PHA with IFN- $\gamma<10 \mathrm{IU} \cdot \mathrm{mL}^{-1}$ could be considered as "possibly altered" rather than "normal". Special attention should be paid to the response to PHA in cases of negative QFT in patients receiving immunosuppressive agents. As the clinical decision to initiate a preventive latent tuberculosis infection treatment before beginning biotherapy relies on QFT, a false negative would lead to an absence of prophylaxis and increased risk of tuberculosis.

Our results must be confirmed by a prospective study aimed at evaluating the risk for false-negative results in low PHA responders [9]. Recent data on QuantiFERON-TB Plus (QFT-Plus; QIAGEN, Hilden, Germany) indicate that QFT-Plus may improve kit performance thanks to the addition of new peptides, aimed at $\mathrm{CD}^{+}{ }^{\mathrm{T}}$-cell stimulation [13]. However, the positive control is based on response to PHA and the global diagnosis strategy remains to be established in immune-compromised individuals.

Julie Belliere ${ }^{1}$ and Antoine Blancher ${ }^{2}$

${ }^{1}$ Nephrology and Organ Transplantation, University Hospital Toulouse, Toulouse Cedex 9, France. ${ }^{2}$ Immunology Laboratory, University Hospital Toulouse, Toulouse, France.

Correspondence: Antoine Blancher, CHU de Toulouse, Immunology, Hopital Rangueil 1 avenue Jean Poulhes, Toulouse 31059, France. E-mail: blancher.a@chu-toulouse.fr

Received: Oct 272016 | Accepted after revision: Dec 152016

Conflict of interest: None declared.

\section{References}

1 Spinicci M, Mencarini J, Goletti D, et al. Discordance between the QuantiFERON-TB Gold In-Tube and Tuberculin Skin Test: Need for a further step? Eur Respir J 2015; 46: 1506-1509.

2 Cirillo DM, Barcellini L, Goletti D. Preliminary data on precision of QuantiFERON-TB Plus performance. Eur Respir J 2016; 48: 955-956.

3 Diel R, Loddenkemper R, Nienhaus A. Evidence-based comparison of commercial interferon-gamma release assays for detecting active TB: a metaanalysis. Chest 2010; 137: 952-968.

4 Helwig U, Müller M, Hedderich J, et al. Corticosteroids and immunosuppressive therapy influence the result of QuantiFERON TB Gold testing in inflammatory bowel disease patients. J Crohns Colitis 2012; 6: 419-424.

5 Dorman SE, Belknap R, Graviss EA, et al. Interferon- $\gamma$ release assays and tuberculin skin testing for diagnosis of latent tuberculosis infection in healthcare workers in the United States. Am J Respir Crit Care Med 2014; 189: 77-87.

6 Woo KS, Kim BG, Choi JL, et al. Neutrophil-to-lymphocyte ratio is associated with impaired interferon-gamma release to phytohemagglutinin. PLoS One 2015; 10: e0125794.

7 Bélard E, Semb S, Ruhwald M, et al. Prednisolone treatment affects the performance of the QuantiFERON gold in-tube test and the tuberculin skin test in patients with autoimmune disorders screened for latent tuberculosis infection. Inflamm Bowel Dis 2011; 17: 2340-2349.

8 Bua A, Ruggeri M, Zanetti S, et al. Effect of teriflunomide on QuantiFERON-TB Gold results. Med Microbiol Immunol 2017; 206: 73-75.

9 Sester M, van Leth F, Bruchfeld J, et al. Risk assessment of tuberculosis in immunocompromised patients. A TBNET Study. Am J Respir Crit Care Med 2014; 190: 1168-1176.

10 Gürol Y, Çelik G. Indeterminate quantiferon TB Gold in Tube results from Turkey. Tuberk Toraks 2016; 64: 182-183.

11 Kobashi Y, Sugiu T, Mouri K, et al. Indeterminate results of QuantiFERON TB-2G test performed in routine clinical practice. Eur Respir J 2009; 33: 812-815.

12 Ferrara G, Losi M, Meacci M, et al. Routine hospital use of a new commercial whole blood interferon-gamma assay for the diagnosis of tuberculosis infection. Am J Respir Crit Care Med 2005; 172: 631-635.

13 Barcellini L, Borroni E, Brown J, et al. First independent evaluation of QuantiFERON-TB Plus performance. Eur Respir J 2016; 47: 1587-1590. 